\title{
ON THE AVERAGE SEPARATION ENERGY OF STRONGLY BOUND NUCLEONS
}

\author{
D. M. BRINK \\ Clarendon Laboratory, oxford ${ }^{\dagger}$ \\ and \\ N. SHERMAN \\ H.M. Randall Laboratory of Physics, \\ University of Michigan, Ann Arbor, Michigan ${ }^{\dagger \dagger}$
}

Received 14 November 1966

\begin{abstract}
The separation energy of a strongly bound nucleon from a light or medium-weight nucleus has been calculated in terms of a general two-body nucleon-nucleon interaction potential. It is assumed that the nuclear wave function can be approximated by a linear combination of a few Slater determinants. Single-particle wave functions are approximated by harmonic oscillator wave functions. Simple formulae, which contain only a few potential matrix elements, are derived for the separation energies and numerical results are presented for several choices of the nucleon-nucleon interaction potential.
\end{abstract}

\section{Introduction}

This paper provides details, improvements and developments of a rough calculation of the separation energy of strongly bound nucleons which was outlined in ref. ${ }^{1}$ ).

The original stimulus for this computation was the interesting $\left(e, e^{\prime} p\right)$ experiments conducted by the group at Sanità ${ }^{2}$ ). At the time when the early results of the Sanità experiments appeared, the effective depth of the shell-model potential for medium nuclei was usually considered ${ }^{t \dagger \dagger}$ to be $\approx 45-50 \mathrm{MeV}\left(\right.$ ref. $\left.^{3}\right)$ ). The $\left(e, \mathrm{e}^{\prime} \mathrm{p}\right)$ measurements indicated that the well depth was greater than $60 \mathrm{MeV}$.

This followed from the argument that if the proton was removed adiabatically, i.e. without disturbing the other nucleons in the nucleus, then the $60 \mathrm{MeV}$ required to remove the most strongly bound group of protons in ${ }^{27} \mathrm{Al}$ (see ref. ${ }^{2}$ )) represents the energy needed to knock a proton out of the lowest occupied shell-model level and the bottom of the potential well is somewhere below this lowest level.

When nucleon separation energies are measured in (e, e'p) or (p, 2p) experiments, the peaks which give the separation energies are broad. In very light nuclei, nucleons ejected from the $\mathrm{p}_{\frac{3}{2}}$ and $\mathrm{p}_{\frac{1}{2}}$ shell-model orbits give rise to separated peaks in the ex-

$\dagger$ This work was supported by the National Science Foundation.

${ }^{\dagger+}$ This work was completed at the Summer Symposium on Nuclear Physics at the University of Michigan, June-July 1966.

$\dagger^{\dagger \dagger}$ (Some earlier calculations of Moszkowski ${ }^{4}$ ) and Skyrme ${ }^{5}$ ) had already indicated that the shellmodel potential might be of the order of $80 \mathrm{MeV}$.). 
perimental spectrum, but in nuclei beyond ${ }^{27} \mathrm{Al}$ these levels are not resolved. It seems therefore appropriate for the theory to predict the average separation energy of a nucleon from these levels. This averaging, as well as making sense experimentally, gives a substantial simplification in the theoretical calculation and leads to a result which depends on fewer parameters.

We hope that our results will be useful to experimentalists performing such experiments, but our own interest is more general. We are interested in computing binding energies in the framework of the shell model. Among the many nucleon-nucleon potentials which lead to agreement with scattering data and properties of nuclear matter, some may not give good results for separation energies. If this turns out to be the case, then computations of separation energies may provide another criterion for selecting among various nucleon-nucleon potentials.

The calculation has several attractive features. The assumptions and approximations are explicit so that refinements can be made if desired. The final formula for the separation energy contains only a few parameters. These few parameters give a good description of many data and it is possible to see which property of the nucleonnucleon interaction determines the separation energy.

Since ref. ${ }^{1}$ ), was published, several Hartree-Fock, self-consistent calculations ${ }^{6}$ ) of nucleon binding energies have been made. Results of these calculations show that harmonic oscillator wave functions are good first approximations to Hartree-Fock wave functions for light, closed-shell nuclei and non-singular, central, nucleonnucleon interactions. In these cases our method gives a good approximation to separation energies obtained from such self-consistent calculations.

The theoretical formulation makes five assumptions.

(i) The nucleon Hamiltonian $H$ describes a system for $A$ nucleons subject to twobody forces

$$
H=T+V=\sum_{i=1}^{A} t_{i}+\frac{1}{2} \sum_{i \neq j}^{A} \sum_{j} v_{i j},
$$

where $t_{i}$ is the kinetic energy of the $i$ th nucleon and $v_{i j}$ the interaction potential of the $i$ th and $j$ th nucleons. If we use the formalism of second quantization, then the Hamiltonian can be written as

$$
H=\sum_{\alpha} \sum_{\beta}\langle\alpha|t| \beta\rangle \eta_{\alpha}^{+} \eta_{\beta}+\frac{1}{4} \sum_{\alpha} \sum_{\beta} \sum_{\gamma} \sum_{\delta}\left\langle\alpha \beta\left|v_{A}\right| \gamma \delta\right\rangle \eta_{\alpha}^{+} \eta_{\beta}^{+} \eta_{\delta} \eta_{\gamma^{\prime}}
$$

where $\left\langle\alpha \beta\left|v_{A}\right| \gamma \delta\right\rangle=\langle\alpha \beta|v| \gamma \delta\rangle-\langle\alpha \beta|v| \delta \gamma\rangle$. The operators $\eta_{\alpha}^{+}$and $\eta_{\alpha}$ are creation and annihilation operators for a set of single nucleon states $|\alpha\rangle$.

(ii) The two-body interaction $v_{i j}$ is charge independent and either sufficiently nonsingular to permit computation of two-particle matrix elements using single-particle wave functions or subject to some treatment which permits computation of matrix elements (e.g. the Moszkowski-Scott ${ }^{7}$ ) separation method).

(iii) The nuclear wave function $\Phi$ can be approximated by a single Slater-deter- 
minant or by a linear combination of Slater determinants subject to the following restrictions:

(a) The single-particle orbital wave functions are taken to be spherical harmonic oscillator wave functions ${ }^{\dagger}$ defined by a potential with harmonic oscillator radius parameter $b=(\hbar / M \omega)^{\frac{1}{2}}$. This parameter is determined by fitting the mean square radius of the nucleus to its experimental value.

(b) The oscillator shell $\rho$ from which the nucleon is removed is completely filled. In other words

$$
\eta_{\alpha}^{+}|\Phi\rangle=0=\langle\Phi| \eta_{\alpha}
$$

for all states $|\alpha\rangle=|n l m \sigma \tau\rangle$ in the oscillator shell $\rho=2 n+l$ ( $n$ is the principal quantum number of the oscillator state, $(I m)$ the orbital angular momentum quantum numbers and $(\sigma \tau)$ the spin and isospin quantum numbers).

(c) In the state $|\Phi\rangle$ there is at most one partially filled oscillator shell for each type of nucleon. The lower oscillator shells are completely filled, the higher ones are completely empty. The coupling scheme of the nucleons in the partially filled shells is arbitrary.

(iv) The separation of the ejected nucleon is accomplished "adiabatically", that is without disturbing any of the nucleons other than the one which is removed. In other words if the initial state of the nucleus is $|\Phi\rangle$ and the nucleon is ejected from a singleparticle state $|\alpha\rangle$, then the final state is $\eta_{\alpha}|\Phi\rangle$.

(v) It is sufficient to retain only that part of the nucleon-nucleon interaction which is a scalar with respect to the symmetry group SU(3) of the harmonic oscillator. We call this the "SU(3) approximation" and we make it for the following reasons:

(a) If we make the $\mathrm{SU}(3)$ approximation then we get a very simple formula for separation energies of strongly bound nucleons.

(b) The corrections to the SU(3) approximation turn out to be relatively small. We investigate these corrections in sect. 6 and calculate them explicitly in several cases. If assumptions (i)-(iv) are valid, then the SU(3) approximation is exact for separation of a nucleon from a closed oscillator shell nucleus.

In sect. 2, we present the formulation of the theory and show how the assumptions enter. We give the final expression for the average adiabatic separation energy $\bar{E}_{\rho}$ from a level $\rho$, but details are discussed in later sections and in appendices. In sect. 3, we evaluate spin sums in the matrix elements, and in sect. 4 , we calculate the orbital parts of the matrix elements. We show in this section how the $\mathrm{SU}(3)$ approximation leads to an especially simple method for computing these separation energies. In sect. 5, we summarize various numerical calculations of matrix elements of the nucleon-nucleon interaction which are given in papers by other workers and which can be used in our formula for the separation energy of $1 \mathrm{~s}$ and $1 \mathrm{p}$ nucleons for nuclei

† The restriction to harmonic oscillator wave functions is used only for the calculation of twoparticle matrix elements of the potential energy and in the "SU(3) approximation" (see assumption (v)). 
with atomic weight $A \leqq 40$. We give a discussion of the validity of two approximations in sect. 6 .

\section{Formalism of the theory}

\subsection{CAlCulation OF THE AVERAGE ADIABATIC SEPARATION ENERGY $\bar{E}_{x}$}

In this section we use the assumptions stated in sect. 1 to obtain an appropriate formula for the average separation energy of a nucleon in an oscillator level $\rho$ of a nucleus. It is convenient to represent the set of quantum numbers ( $n \ln \sigma \tau)$ by a single Greek letter $\alpha, \beta$, etc. The initial nuclear state is $|\Phi\rangle$. If we remove a nucleon from the single-particle state $|\alpha\rangle$, then the final nuclear state is $\eta_{\alpha}|\Phi\rangle$ [assumption (iv)]. The adiabatic separation energy $E_{\alpha}$ of a nucleon from the state $|\alpha\rangle$ is given by the difference in energies of the initial and final states

$$
\begin{aligned}
\left(-E_{\alpha}\right) & =\langle\Phi|(T+V)| \Phi\rangle-\left\langle\Phi\left|\eta_{\alpha}^{+}(T+V) \eta_{\alpha}\right| \Phi\right\rangle \\
& =\left\langle\Phi\left|(T+V)-\eta_{\alpha}^{+}(T+V) \eta_{\alpha}\right| \Phi\right\rangle .
\end{aligned}
$$

We show in appendix 1 that the operator whose expectation value is taken in eq. (2.1) may be transformed to

$$
T+V-\eta_{\alpha}^{+}(T+V) \eta_{x}=\langle\alpha|t| \alpha\rangle+W_{\alpha}+\eta_{\alpha} C+D \eta_{\alpha}^{+}-\eta_{x}(T+V) \eta_{x}^{+}
$$

where $C$ and $D$ are certain operators whose exact form is not important, and a singleparticle operator $W_{\alpha}$ is defined by

$$
W_{\alpha}=\sum_{\beta} \sum_{\gamma}\left\langle\alpha \beta\left|v_{A}\right| \alpha \gamma\right\rangle \eta_{\beta}^{+} \eta_{\gamma}
$$

If we substitute eq. (2.2), into eq. (2.1) and use assumption (iiib), then the last two terms of (2.2) make no contribution to the expectation value and we get

$$
\left(-E_{\alpha}\right)=\langle\alpha|t| \alpha\rangle+\left\langle\Phi\left|W_{\alpha}\right| \Phi\right\rangle .
$$

In order to find the average separation energy $\bar{E}_{\rho_{1}}$ of a nucleon from an oscillator shell $\rho_{1}$, we would average over the orbital, spin and isospin quantum numbers of all single-particle states in that shell.

By averaging over the isospin quantum number $\tau$, we obtain the mean separation energy of a nucleon, either a proton or neutron. If we want to find the separation energy of a proton we should not average over $\tau$ but must put $\tau=\frac{1}{2}$. However, if nuclear forces are charge independent, then proton and neutron separation energies are almost equal (but not exactly equal since a proton, ejected from a nucleus with unequal numbers of protons and neutrons, can interact with neutrons in more states than can a corresponding neutron) so that averaging over $\tau$ leads to the average nucleon separation energy. In addition, the Coulomb energy makes an appreciable difference to the proton separation energy but this can be added explicitly at the end of the calculation. 
Let us introduce an "average interaction operator" for the level $\rho_{1}$ defined by

$$
W\left(\rho_{1}\right)=\sum_{\beta} \sum_{\gamma} \eta_{\beta}^{+} \eta_{\gamma}\left\langle\beta\left|W_{\rho_{1}}\right| \gamma\right\rangle
$$

The matrix elements

$$
\left\langle\beta\left|W_{\rho_{1}}\right| \gamma\right\rangle=\frac{1}{4\left[\rho_{1}\right]} \sum_{\alpha}^{\left(\rho_{1}\right)}\left\langle\beta \alpha\left|v_{A}\right| \gamma \alpha\right\rangle
$$

where the sum is taken over all single-nucleon states $|\alpha\rangle=|n| m \sigma \tau\rangle$ belonging to the oscillator shell $\rho_{1}\left(2 n+l=\rho_{1}\right)$. The quantity

$$
\left[\rho_{1}\right]=\frac{1}{2}\left(\rho_{1}+1\right)\left(\rho_{1}+2\right)
$$

stands for the orbital degeneracy of the oscillator level $\rho_{1}$. Then we obtain the desired expression for the average separation energy of a neutron from the oscillator level $\rho_{1}$

$$
\left(-\bar{E}_{\rho_{1}}\right)=\left\langle\rho_{1}|t| \rho_{1}\right\rangle+\left\langle\Phi\left|W\left(\rho_{1}\right)\right| \Phi\right\rangle
$$

The kinetic energy matrix element is

$$
\left\langle\rho_{1}|t| \rho_{1}\right\rangle=\frac{1}{4}\left(2 \rho_{1}+1\right) h \omega=\frac{1}{4}\left(2 \rho_{1}+1\right)\left(\frac{h^{2}}{M b^{2}}\right) .
$$

\subsection{PROPERTIES OF THE SINGLE-PARTICLE OPERATOR $W\left(\rho_{1}\right)$}

The operator $W\left(\rho_{1}\right)$ is defined by its single-particle matrix elements [eq. (2.6)]

$$
\begin{aligned}
& \left\langle n_{2} l_{2} m_{2} \sigma_{2} \tau_{2}\left|W_{\rho_{1}}\right| n_{2}^{\prime} l_{2}^{\prime} m_{2}^{\prime} \sigma_{2}^{\prime} \tau_{2}^{\prime}\right\rangle \\
& =\frac{1}{4\left[\rho_{1}\right]} \sum_{n_{1} l_{1} m_{1} \sigma_{1} \tau_{1}}\left\langle n_{2} l_{2} m_{2} \sigma_{2} \tau_{2} ; n_{1} l_{1} m_{1} \sigma_{1} \tau_{1}\left|v_{A}\right| n_{2}^{\prime} l_{2}^{\prime} m_{2}^{\prime} \sigma_{2}^{\prime} \tau_{2}^{\prime} ; n_{1} l_{1} m_{1} \sigma_{1} \tau_{1}\right\rangle .
\end{aligned}
$$

The antisymmetrized nucleon-nucleon interaction $v_{A}$ is a scalar in ordinary space. If the interaction is charge independent it is also a scalar in isospin space. Since the definition of $W\left(\rho_{1}\right)$ involves an average over all spins, isospin and orbital magnetic substates of the level $\rho_{1}$, it follows that $W\left(\rho_{1}\right)$ has the same tensor character as $v_{A}$ in orbital, spin and isospin space. We will discuss certain special cases in detail.

2.2.1. Central force. A central force is a scalar in spin and space orbital space. The operator $W\left(\rho_{1}\right)$ has the same character and its matrix elements are therefore diagonal in $l_{2} m_{2} \sigma_{2} \tau_{2}$ and independent of $m_{2} \sigma_{2} \tau_{2}$, i.e.

$$
\begin{aligned}
& \left\langle n_{2} l_{2} m_{2} \sigma_{2} \tau_{2}\left|W_{\rho_{1}}^{\mathrm{c}}\right| n_{2}^{\prime} l_{2}^{\prime} m_{2}^{\prime} \sigma_{2}^{\prime} \tau_{2}^{\prime}\right\rangle \\
& \quad=\delta\left(l_{2} l_{2}^{\prime}\right) \delta\left(m_{2} m_{2}^{\prime}\right) \delta\left(\sigma_{2} \sigma_{2}^{\prime}\right) \delta\left(\tau_{2} \tau_{2}^{\prime}\right)\left\langle n_{2} l_{2}\left|W_{\rho_{1}}\right| n_{2}^{\prime} l_{2}\right\rangle
\end{aligned}
$$

2.2.2. Spin-orbit force. A spin-orbit force is a vector in spin space and orbital space; but is a scalar for a combined spin and space rotation. Hence the matrix ele- 
ments of $W_{\rho_{1}}$ will not be diagonal in $m_{1}, \sigma_{1}$ but will be diagonal only in $j$ and $m_{j}$

$$
\begin{aligned}
& \left\langle n_{2} l_{2} j_{2} m_{j_{2}} \tau_{2}\left|W_{\rho_{1}}^{\text {so }}\right| n_{2}^{\prime} l^{\prime} j_{2}^{\prime} m_{j_{2}}^{\prime} \tau_{2}^{\prime}\right\rangle \\
& =\delta\left(l_{2} l_{2}^{\prime}\right) \delta\left(j_{2} j_{2}^{\prime}\right) \delta\left(m_{j_{2}} m_{j_{2}}^{\prime}\right) \delta\left(\tau_{2} \tau_{2}^{\prime}\right)\left\langle n_{2} l_{2} j_{2}\left|W_{\rho_{1}}\right| n_{2}^{\prime} l_{2} j_{2}\right\rangle .
\end{aligned}
$$

2.2.3. Tensor force. A tensor force is a tensor of rank two in spin space and orbital space but is a scalar for a combined spin and space rotation. The matrix elements of $W_{\rho_{1}}$ will have the same tensor character. However the matrix elements of $W_{\rho_{1}}$ are taken between states with spin $\frac{1}{2}$ and the spin part of $W_{\rho_{t}}$ which is of rank 2 cannot connect two such states. Hence the matrix elements of $W_{\rho_{1}}$ for the tensor force are zero.

2.2.4. The SU(3) approximation. If the single-particle wave functions in the state $|\Phi\rangle$ are harmonic oscillator wave functions, then it is useful to consider the nucleonnucleon interaction as a combination of tensors of the symmetry group SU(3) of the harmonic oscillator ${ }^{9}$ ). It may be a good approximation to calculate separation energies using only that part $v_{A}^{0}$ of the nucleon-nucleon interaction which is a scalar of SU(3). The corresponding part of the single-particle operator $W_{\rho}^{0}$ will also be a scalar in SU(3) and will therefore be diagonal in all the quantum numbers $n_{2} l_{2} m_{2} \sigma_{2} \tau_{2}$

$$
\begin{aligned}
\left\langle n_{2} l_{2} m_{2} \sigma_{2} \tau_{2}\left|W_{\rho_{1}}^{0}\right| n_{2}^{\prime} l_{2}^{\prime} m_{2}^{\prime} \sigma_{2}^{\prime} \tau_{2}^{\prime}\right\rangle & \\
& =\delta\left(n_{2} n_{2}^{\prime}\right) \delta\left(l_{2} l_{2}^{\prime}\right) \delta\left(m_{2} m_{2}^{\prime}\right) \delta\left(\sigma_{2} \sigma_{2}^{\prime}\right) \delta\left(\tau_{2} \tau_{2}^{\prime}\right)\left\langle\rho_{2}\left|W_{\rho_{1}}^{0}\right| \rho_{2}\right\rangle .
\end{aligned}
$$

\subsection{FINAL FORM OF THE SEPARATION ENERGY}

Before we turn to the details of the calculation of separation energies, we will write down the final formulae and discuss the significance of the various terms. In subsect. 2.2., we showed that tensor forces do not contribute to separation energies if we make a calculation based on the assumptions stated in sect. 1 . Spin-orbit forces can contribute; but we will not consider them in this section and postpone a discussion of their effects until sect. 6. Here we will discuss the resulting $\left(\bar{E}_{\rho}\right)$ under conditions that only central forces make a significant contribution.

2.3.1. Without the SU(3) approximation. In subsect. 2.2 [see eq. (2.11)], we showed that the operator $W\left(\rho_{1}\right)$ is diagonal in the angular momentum spin and isospin quantum numbers. If we substitute eq. (2.11) into eq. (2.5), we find that

$$
W\left(\rho_{1}\right)=\sum_{n^{\prime} n_{2} l_{2} m_{2} \sigma_{2} \tau_{2}} \eta_{n^{\prime} l_{2} m_{2} \sigma_{2} \tau_{2}}^{+} \eta_{n_{2} l_{2} m_{2} \sigma_{2} \tau_{2}}^{+}\left\langle n_{2} l_{2}\left|W_{\rho_{1}}\right| n_{2}^{\prime} l_{2}\right\rangle \text {. }
$$

Substituting in eq. (2.8), we get for the separation energy $\bar{E}_{p_{1}}$

$$
-\bar{E}_{\rho_{1}}=\left\langle\rho_{1}|t| \rho_{1}\right\rangle+\sum_{n^{\prime}{ }_{2} n_{2} l_{2}}\left\langle\Phi\left|\sum_{m_{2} \sigma_{2} \tau_{2}}\left(\eta_{n^{\prime}{ }_{2} l_{2} m_{2} \sigma_{2} \tau_{2}}^{+} \eta_{n_{2} l_{2} m_{2} \sigma_{2} \tau_{2}}\right)\right| \Phi\right\rangle\left\langle n_{2}^{\prime} l_{2}\left|W_{\rho_{1}}\right| n_{2} l_{2}\right\rangle .
$$

It follows from assumption (iiic) described in sect. 1 that only terms with $n_{2}^{\prime}=n_{2}$ contribute. The expectation value in the last equation is

$$
\bar{N}_{n_{2} l_{2}}=\left\langle\Phi\left|\sum_{m_{2} \sigma_{2} \tau_{2}}\left(\eta_{n_{2} l_{2} m_{2} \sigma_{2} \tau_{2}}^{+} \eta_{n_{2} l_{2} m_{2} \sigma_{2} \tau_{2}}\right)\right| \Phi\right\rangle
$$


which is just the expectation value of the number of nucleons in the orbital state $\left(n_{2} l_{2}\right)$. Hence the formula for the separation energy is

$$
\left(-\bar{E}_{\rho_{1}}\right)=\left\langle\rho_{1}|t| \rho_{1}\right\rangle+\sum_{n_{2} l_{2}} \bar{N}_{n_{2} l_{2}}\left\langle n_{2} l_{2}\left|W_{\rho_{1}}\right| n_{2} l_{2}\right\rangle .
$$

In sect. 4 , eq. (4.5), we show that the matrix element of $W_{\rho_{1}}$ which appears in eq. (2.16) can be calculated in terms of coefficients $V_{n l}$ of eq. (4.4) which determine the average (central) interaction energy of two nucleons in a state of relative motion characterized by quantum numbers $(n l)$

$$
\begin{aligned}
& \left\langle n_{2} l_{2}\left|W_{\rho_{1}}\right| n_{2} l_{2}\right\rangle \\
& \quad=\frac{1}{(2 l+1)\left[\rho_{1}\right]} \sum_{\substack{n l N L \lambda \\
\left(2 n_{1}+l_{1}=\rho_{1}\right)}}(2 \lambda+1)\left|\left\langle n_{1} l_{1} n_{2} l_{2} \lambda \mid n l N L \lambda\right\rangle\right|^{2} V_{n l}\left[1-\frac{1}{4}(-1)^{2}\right],
\end{aligned}
$$

where the sum is taken over all values of $n l N L \lambda$ and over values of $n_{1} l_{1}$ which satisfy the restriction $2 n_{1}+l_{1}=\rho_{1}$. The quantities $\left\langle n_{1} l_{1} n_{2} l_{2} \lambda \mid n l N L \lambda\right\rangle$ are the Moshinsky transformation coefficients which are tabulated in ref. ${ }^{8}$ ). If the interaction strengths $V_{n l}$ are given, eqs. (2.16) and (2.17) may be used to calculate the separation energy from the oscillator shell $\rho_{1}$.

An interesting feature of the result is that the separation energy is independent of the details of the coupling of nucleons in the last unfilled shell and depends only on the average occupation numbers $\bar{N}_{n_{2} l_{2}}$ of the orbital states.

2.3.2. With the SU(3) approximation. Eqs. (2.16) and (2.17) give a correct formula for the separation energies of strongly bound nucleons interacting through central (or central plus tensor) forces. However eq. (2.17) is rather complicated and the evaluation of the matrix element $\left\langle n_{2} l_{2}\left|W_{\rho_{1}}\right| n_{2} l_{2}\right\rangle$ requires the use of extensive tables of Mosbinsky coefficients. We can obtain a simple result which is at the same time almost as accurate as eqs. (2.16) and (2.17) by making the SU(3) approximation, i.e. by retaining only that part of the nucleon-nucleon interaction which is a scalar with respect to SU(3) [assumption (vi)].

We showed in subsect. 2.2 that the SU(3) scalar part $W_{\rho_{1}}^{0}$ of the operator $W_{\rho_{1}}$ is diagonal in all quantum numbers. If we use this result the formula for the separation energy analogous to eq. (2.16) is

$$
\left(-\bar{E}_{\rho_{1}}\right)=\left\langle\rho_{1}|t| \rho_{1}\right\rangle+\sum_{\rho_{2}} \bar{N}_{\rho_{2}}\left\langle\rho_{2}\left|W_{\rho_{1}}\right| \rho_{2}\right\rangle,
$$

where $\bar{N}_{\rho_{2}}$ is the number of nucleons in the oscillator shell $\rho_{2}$. Note that we do not need to use assumption (iiic) to derive eq. (2.18), if we use the SU(3) approximation.

In sect. 4 , we calculate the matrix element $\left\langle\rho_{2}\left|W_{\rho_{1}}\right| \rho_{2}\right\rangle$ appearing in eq. (2.18) in terms of quantities $V_{\rho_{1}}$ [eq. (4.7a)] which determine the average interaction of two nucleons in a state of relative motion with total oscillator quantum number $2 n_{1}+l_{1}$ $=\rho_{1}$. The result is

$$
\left\langle\rho_{2}\left|W_{\rho_{1}}\right| \rho_{2}\right\rangle=\sum_{\rho} \sum_{P} W\left(\rho_{1} \rho_{2}, \rho P\right) V_{\rho}\left(1-\frac{1}{4}(-1)^{\rho}\right) .
$$


The coefficients $W\left(\rho_{1} \rho_{2}, \rho P\right)$ are defined in eq. (4.9) and given in table 1 for $\rho_{1}, \rho_{2} \leqq 3$. Explicit recursion relations are also given in sect. 4 . These may be used to calculate the coefficients for other values of $\rho_{1}$ and $\rho_{2}$ if required.

Eq. (2.18) shows that the separation energy of a strongly bound nucleon is independent of the coupling of nucleons in unfilled shells. The contribution of nucleons in unfilled oscillator shells is proportional to the number of nucleons in those shells. In ref. ${ }^{1}$ ), we assumed this property. Here we have shown that it is a consequence of the SU(3) approximation.

\section{Evaluation of spin sums for central forces}

In order to use eq. (2.16) for the separation energy, it is necessary to evaluate the matrix elements

$$
\begin{aligned}
& \left\langle n_{2} l_{2}\left|W_{\rho_{1}}\right| n_{2} l_{2}\right\rangle \\
& =\frac{1}{4\left[\rho_{1}\right]} \sum_{\substack{n_{1} l_{1} m_{1} \\
\sigma_{1} \tau_{1}}}^{\left(\rho_{1}\right)}\left\langle n_{2} l_{2} m_{2} \sigma_{2} \tau_{2}, n_{1} l_{1} m_{1} \sigma_{1} \tau_{1}\left|v_{A}\right| n_{2} l_{2} m_{2} \sigma_{2} \tau_{2}, n_{1} l_{1} m_{1} \sigma_{1} \tau_{1}\right\rangle .
\end{aligned}
$$

For a general central interaction, we can write it in terms of its singlet and triplet parts

$$
v=V_{s} \frac{\left(1-P_{\sigma}\right)}{2}+V_{t} \frac{\left(1+P_{\sigma}\right)}{2},
$$

where $P_{\sigma}$ is the usual spin-exchange operator and $V_{s}$ and $V_{t}$ appropriate (possibly non-local) functions of the relative spatial coordinates of the two nucleons. The matrix elements in (3.1) contain the exchange parts which are included in the antisymmetrized interaction operator $v_{\boldsymbol{A}}$. This can be written as

$$
v_{A}=v\left(1-P_{M} P_{\sigma} P_{\tau}\right)
$$

where $P_{M}$ and $P_{\tau}$ are the usual space and isospin exchange operators, respectively. The average value of $P_{\sigma}$ averaged over spins and of $P_{\tau}$ averaged over isospin is $\frac{1}{2}$, e.g.

$\frac{1}{4} \sum_{\sigma_{1}} \sum_{\sigma_{2}}\left\langle\sigma_{1} \sigma_{2}\left|P_{\sigma}\right| \sigma_{1} \sigma_{2}\right\rangle=\frac{1}{4} \sum_{\sigma_{1}} \sum_{\sigma_{2}}\left\langle\sigma_{1} \sigma_{2} \mid \sigma_{2} \sigma_{1}\right\rangle=\frac{1}{4} \sum_{\sigma_{1}} \sum_{\sigma_{2}} \dot{\delta}_{\sigma_{1} \sigma_{2}}=\frac{1}{4} \sum_{\sigma_{1}} 1=\frac{1}{2}$.

We can use these properties to simplify (3.1) by noting that $\left\langle n_{2} l_{2}\left|W_{\rho_{1}}\right| n_{2} l_{2}\right\rangle$ does not depend on $m_{2}, \sigma_{2}$, or $\tau_{2}$. We can therefore write

$$
\begin{aligned}
& \left\langle n_{2} l_{2}\left|W_{\rho_{1}}\right| n_{2} l_{2}\right\rangle=\frac{1}{4\left(2 l_{2}+1\right)} \sum_{m_{2}} \sum_{\sigma_{2}} \sum_{\tau_{2}}\left\langle n_{2} l_{2}\left|W_{\rho_{1}}\right| n_{2} l_{2}\right\rangle \\
& =\frac{1}{16\left(2 l_{2}+1\right)\left[\rho_{1}\right]} \sum_{n_{1} l_{1}}^{\left.\rho_{1}\right)} \sum_{\substack{m_{1} \sigma_{1} \tau_{1} \\
m_{2} \sigma_{2} \tau_{2}}}\left\langle n_{2} l_{2} m_{2} \sigma_{2} \tau_{2}, n_{1} l_{1} m_{1} \sigma_{1} \tau_{1}\left|v_{A}\right| n_{2} l_{2} m_{2} \sigma_{2} \tau_{2}, n_{1} l_{1} m_{1} \sigma_{1} \tau_{1}\right\rangle
\end{aligned}
$$


and make use of the separability properties of the central force to evaluate the spin and isospin sums. That is, we can separate parts of the matrix elements (in (3.4)) containing $V_{s}$ to $V_{t}$ and exchange operators into products of matrix elements e.g.

$$
\begin{aligned}
& \left\langle n_{2} l_{2} m_{2} \sigma_{2} \tau_{2}, n_{1} l_{1} \sigma_{1} \tau_{1}\left|V_{\mathrm{s}} P_{n} P_{\sigma} P_{\tau}\right| n_{2} l_{2} m_{2} \sigma_{2} \tau_{2}, n_{1} l_{1} m_{1} \sigma_{1} \tau_{1}\right\rangle \\
& \quad=\left\langle n_{2} l_{2} m_{2}, n_{1} l_{1} m_{1}\left|V_{\mathrm{s}} P_{n}\right| n_{2} l_{2} m_{2}, n_{1} l_{1} m_{1}\right\rangle\left\langle\sigma_{2} \sigma_{1}\left|P_{\sigma}\right| \sigma_{2} \sigma_{1}\right\rangle\left\langle\tau_{2} \tau_{1}\left|P_{\mathrm{z}}\right| \tau_{2} \tau_{1}\right\rangle .
\end{aligned}
$$

We can then average over $\sigma_{1}, \sigma_{2}, \tau_{1}$ and $\tau_{2}$ as in eq. (3.3). Writing

$$
\begin{aligned}
& V_{\mathrm{s}}\left(\frac{1-P_{\sigma}}{2}\right)\left(1-P_{M} P_{\sigma} P_{\tau}\right)=\frac{1}{2} V_{\mathrm{s}}\left(1-P_{\sigma}-P_{M} P_{\sigma} P_{\tau}+P_{M} P_{\tau}\right), \\
& V_{\mathrm{t}}\left(\frac{1+P_{\sigma}}{2}\right)\left(1-P_{M} P_{\sigma} P_{\tau}\right)=\frac{1}{2} V_{\mathrm{t}}\left(1+P_{\sigma}-P_{M} P_{\sigma} P_{\tau}-P_{M} P_{\tau}\right),
\end{aligned}
$$

then separating the matrix elements as above and summing, we obtain

$$
\begin{aligned}
\frac{1}{16} \sum_{\sigma_{1}} \sum_{\sigma_{2}} \sum_{\tau_{1}} \sum_{\tau_{2}}\left\langle n_{2} l_{2} m_{2} \sigma_{2} \tau_{2}, n_{1} l_{1} m_{1} \sigma_{1} \tau_{1}\left|v_{A}\right| n_{2} l_{2} m_{2} \sigma_{2} \tau_{2}, n_{1} l_{1} m_{1} \sigma_{1} \tau_{1}\right\rangle \\
\quad=\left\langle n_{2} l_{2} m_{2} n_{1} l_{1} m_{1}\left|\left\{V_{\mathrm{s}}\left(\frac{1}{4}+\frac{1}{8} P_{M}\right)+V_{\mathrm{t}}\left(\frac{3}{4}-\frac{3}{8} P_{M}\right)\right\}\right| n_{2} l_{2} m_{2} n_{1} l_{1} m_{1}\right\rangle \\
=\left\langle n_{2} l_{2} m_{2} n_{1} l_{1} m_{1}\left|\bar{v}_{A}\right| n_{2} l_{2} m_{2} n_{1} l_{1} m_{1}\right\rangle .
\end{aligned}
$$

In other words we can replace $v_{A}$ in eq. (3.4) by the equivalent

$$
\bar{v}_{A}=V_{\mathrm{s}}\left(\frac{1}{4}+\frac{1}{8} P_{M}\right)+V_{\mathrm{t}}\left(\frac{3}{4}-\frac{3}{8} P_{M}\right) \text {. }
$$

Further, in an even orbital state $\left(P_{M}=1\right)$ so that

$$
\left(\bar{v}_{A}\right)_{\text {even }}=\frac{3}{4}\left(\frac{1}{2} V_{\mathrm{s}}+\frac{1}{2} V_{\mathrm{t}}\right)
$$

and in an odd orbital state $\left(P_{M}=-1\right)$ so that

$$
\left(\bar{v}_{A}\right)_{\text {odd }}=\frac{5}{4}\left(\frac{1}{10} V_{\mathrm{s}}+\frac{9}{10} V_{\mathrm{t}}\right)
$$

These results may be summarized as follows:

The arbitrary spin-dependent central nucleon-nucleon interaction $v$ in eq. (3.2) can be replaced in eq. (3.4) by the spin-independent interaction

$$
\bar{v}= \begin{cases}\frac{1}{2}\left(V_{\mathrm{s}}+V_{\mathrm{t}}\right) & \text { in even-orbital states } \\ \frac{1}{10}\left(V_{\mathrm{s}}+q V_{\mathrm{t}}\right) & \text { in odd-orbital states, }\end{cases}
$$

and $v_{A}$ can be replaced by

$$
\bar{v}_{A}=\bar{v}\left(1-\frac{1}{4} P_{M}\right)
$$

in evaluating the matrix elements of $W_{\rho_{1}}$.

The "average interaction" $\bar{v}$ is the scalar part ${ }^{9}$ ) of the interaction $v$ with respect to the supermultiplet symmetry group. The separation energy depends only on these 
averages of the singlet and triplet interactions because we have averaged over the spin and isospin of the ejected nucleon.

If we use $\bar{v}_{A}$, then eq. (3.4) reduces to

$$
\left\langle n_{2} l_{2}\left|W_{\rho_{1}}\right| n_{2} l_{2}\right\rangle=\frac{1}{\left(2 l_{2}+1\right)\left[\rho_{1}\right]} \sum_{n_{1} l_{1}}^{\left(\rho_{1}\right)} \sum_{m_{1}} \sum_{m_{2}}\left\langle n_{2} l_{2} m_{2} n_{1} l_{1} m_{1}\left|\bar{v}_{A}\right| n_{2} l_{2} m_{2} n_{1} l_{1} m_{1}\right\rangle
$$

or for matrix elements between states with total orbital angular momentum $\lambda$

$$
\begin{aligned}
\sum_{m_{1}} \sum_{m_{2}}\left\langle n_{2} l_{2} m_{2} n_{1} l_{1} m_{1}\left|\vec{v}_{A}\right| n_{2} l_{2} m_{2} n_{1} l_{1} m_{1}\right\rangle & =\sum_{\lambda \mu}\left\langle n_{2} l_{2} n_{1} l_{1} \hat{\lambda} \mu\left|\bar{v}_{A}\right| n_{2} l_{2} n_{1} l_{1} \lambda \mu\right\rangle \\
& =\sum_{\lambda}(2 \lambda+1)\left\langle n_{2} l_{2} n_{1} l_{1} \lambda\left|\bar{v}_{A}\right| n_{2} l_{2} n_{1} l_{1} \lambda\right\rangle,
\end{aligned}
$$

( since $\bar{v}_{\boldsymbol{A}}$ is a scalar) and

$$
\left\langle n_{2} l_{2}\left|W_{\rho_{1}}\right| n_{2} l_{2}\right\rangle=\frac{1}{\left(2 l_{2}+1\right)\left[\overline{\left.\rho_{1}\right]}\right.} \sum_{n_{1} l_{1}}^{\left(\rho_{1}\right)} \sum_{\lambda}(2 \lambda+1)\left\langle n_{2} l_{2} n_{1} l_{1} \hat{\lambda}\left|\bar{v}_{A}\right| n_{2} l_{2} n_{1} l_{1} \lambda\right\rangle .
$$

\section{Evaluation of orbital matrix elements}

Formulae (2.16) and (2.18) for the separation energy $\bar{E}_{\rho_{1}}$ can be simplified using Talmi-Moshinsky ${ }^{8}$ ) techniques for expressing two-nucleon wave functions in terms of centre-of-mass and relative motion wave functions. We will discuss this reduction explicitly for two cases. In case (i) we neglect spin-orbit coupling and call this "the central force approximation" (we have seen that the tensor force does not contribute), and in case (ii) we also make the $\mathrm{SU}(3)$ approximation.

\subsection{THE CENTRAL FORCE APPROXIMATION}

The matrix element $\left\langle n_{2} l_{2}\left|W_{p_{1}}\right| n_{2} l_{2}\right\rangle$ appearing in eq. (3.7) is expressed in terms of the average spin-independent, nucleon-nucleon interaction $\bar{v}_{A}$ given by eq. (3.5). In eq. (3.7), the state $\left|n_{1} l_{1} n_{2} l_{2} \lambda\right\rangle$ describes two nucleons in the harmonic oscillator orbital states $\left(n_{1} l_{1}\right)\left(n_{2} l_{2}\right)$ with their orbital angular momenta coupled to a resultant $\lambda$. This state may be expressed in terms of relative-motion and centre-of-mass states using the Moshinsky coefficients

$$
\left.\left|n_{2} l_{2} n_{1} l_{1} \lambda\right\rangle=\sum_{n l N L}|n| N L \lambda\right\rangle\left\langle n|N L \lambda| n_{2} l_{2} n_{1} l_{1} \lambda\right\rangle
$$

where $n l$ and $N L$ are the quantum numbers characterizing the relative and centre-ofmass motion of the two nucleons. We can use eq. (4.1) to transform the matrix element in eq. (3.7)

$$
\begin{aligned}
\left\langle n_{2} l_{2} n_{1} l_{1} \lambda\left|\bar{v}_{A}\right| n_{2} l_{2} n_{1} l_{1} \lambda\right\rangle & \\
& =\sum_{n^{\prime} n I N L}\left\langle n_{2} l_{2} n_{1} l_{1} \lambda|n| N L \lambda\right\rangle\left\langle n^{\prime} l N L \lambda \mid n_{2} l_{2} n_{1} l_{1} \lambda\right\rangle\left\langle n l\left|\bar{v}_{A}\right| n^{\prime} l\right\rangle .
\end{aligned}
$$


We must have $n=n^{\prime}$ in eq. (4.2) because a Moshinsky coefficient is zero unless $2 n_{2}+l_{2}+2 n_{1}+l_{1}=2 n+l+2 N+L$. Also from eq. (3.5), we have

$$
\left\langle n l\left|\bar{v}_{A}\right| n l\right\rangle=\left(1-\frac{1}{4}(-1)^{l}\right) V_{n l},
$$

where

$$
V_{n l}=\langle n l|\bar{v}| n l\rangle \text {. }
$$

Substituting eqs. (4.2) and (4.3) into eq. (3.7), we get

$$
\left\langle n_{2} l_{2}\left|W_{\rho_{1}}\right| n_{2} l_{2}\right\rangle=\frac{1}{\left(2 l_{2}+1\right)\left[\rho_{1}\right]} \sum_{\substack{n l N L \\ \lambda n_{1} l_{1}}}(2 \lambda+1)\left\langle n_{2} l_{2} n_{1} l_{1} \lambda \mid n l N L \lambda\right\rangle^{2}\left(1-\frac{1}{4}(-1)^{l}\right) V_{n l}
$$

The quantity $V_{n l}$ defined in eq. (4.4) is the average interaction strength for two nucleons in a state of relative motion characterized by the quantum numbers $n l$.

\subsection{THE SU(3) APPROXIMATION}

If we make the SU(3) approximation then only that part $\bar{v}$ of the average nucleonnucleon interaction $\bar{v}$ which is a scalar with respect to the harmonic oscillator symmetry group SU(3) contributes to the separation energy. It is convenient to use the methods of ref. ${ }^{9}$ ) and expand this part of the interaction as

$$
\bar{v}=\sum_{\rho} V_{\rho} q_{\rho}
$$

where the coefficients $V_{\rho}$ are linear combinations of the coefficients $V_{n l}$ with $2 n+l=\rho$, i.e.

$$
V_{\rho}=\frac{1}{[\rho]} \sum_{2 n+l=\rho}(2 l+1) V_{n l},
$$

and $q_{\rho}$ is a projection operator on to these states of relative motion of the two interacting nucleons with total oscillator quantum number equal to $\rho$, i.e.

$$
\left\langle\rho^{\prime} u\left|q_{\rho}\right| \rho^{\prime \prime} v\right\rangle=\delta_{\rho \rho^{\prime}} \delta_{\rho \rho^{\prime \prime}} \delta_{u v}
$$

where $u$ and $v$ stand for quantum numbers which distinguish states with the same total oscillator quantum number. For example $u$ could stand for the quantum numbers $\mathrm{lm}$ or for quantum numbers $n_{\mathrm{x}} n_{\mathrm{y}} n_{\mathrm{z}}$ which would be appropriate when discussing oscillator wave functions in a rectangular coordinate system. In the $\mathrm{SU}(3)$ approximation, the quantity analogous to $\left\langle n_{2} l_{2}\left|W_{\rho_{1}}\right| n_{2} l_{2}\right\rangle$ in eq. (3.6) is

$$
\left\langle\rho_{2}\left|W_{\rho_{1}}^{0}\right| \rho_{2}\right\rangle=\frac{1}{\left[\rho_{1}\right]\left[\rho_{2}\right]} \sum_{u_{1}} \sum_{u_{2}}\left\langle\rho_{2} u_{2} \rho_{1} u_{1}\left|\vec{v}_{A}\right| \rho_{2} u_{2} \rho_{1} u_{1}\right\rangle,
$$

where the zero superscript indicates an $\mathrm{SU}(3)$ scalar and $\vec{v}_{A}$ is given by the analogue of eq. (3.5) with $\bar{v}$ replaced by $\bar{v}$ defined in eq. (4.6), i.e.

$$
\bar{v}_{A}=\tilde{v}\left(1-\frac{1}{4} P_{M}\right) \text {. }
$$


Substituting for $\tilde{v}_{A}$, using eq. (4.6), we have

where

$$
\left\langle\rho_{2}\left|W_{\rho_{1}}^{0}\right| \rho_{2}\right\rangle=\sum_{\rho} \sum_{P} V_{\rho}\left(1-\frac{1}{4}(-1)^{\rho}\right) W\left(\rho_{2} \rho_{1}, \rho P\right)
$$

$$
W\left(\rho_{2} \rho_{1}, \rho P\right)=\frac{1}{\left[\rho_{1}\right]\left[\rho_{2}\right]} \sum_{u_{1}} \sum_{u_{2}}\left\langle\rho_{2} u_{2} \rho_{1} u_{1}\left|q_{\rho}\right| \rho_{2} u_{2} \rho_{1} u_{1}\right\rangle .
$$

In eq. (4.8), the quantities $V_{\rho}$ depend on the nucleon-nucleon interaction while $W\left(\rho_{2} \rho_{1}, \rho P\right)$ are numerical coefficients which are independent of the nucleon-nucleon interaction and depend only on the quantum numbers $\rho_{1}, \rho_{2}$ and $\rho$. The centre-ofmass oscillator quantum numbers $P=\rho_{1}+\rho_{2}-\rho$ is included in the coefficient $W$ in order to simplify its symmetry properties.

We will list certain properties of the coefficients $W\left(\rho_{2} \rho_{1}, \rho P\right)$ which are sufficient to determine them. Table 1 gives their numerical values for $\rho_{1}, \rho_{2} \leqq 3$.

TABLE 1

Values of the coefficients $W\left(\rho_{1} \rho_{2}, \rho P\right)$ for $\rho_{1}, \rho_{2} \leqq 3,\left(\rho_{1}+\rho_{2}=\rho-P\right)$

\begin{tabular}{cccccccc}
\hline & 0 & 1 & 2 & 3 & 4 & 5 & 6 \\
\hline 00 & 1 & 0 & 0 & 0 & 0 & 0 & 0 \\
10 & $\frac{1}{2}$ & $\frac{1}{2}$ & 0 & 0 & 0 & 0 & 0 \\
20 & $\frac{1}{4}$ & $\frac{1}{2}$ & $\frac{1}{4}$ & 0 & 0 & 0 & 0 \\
30 & $\frac{1}{8}$ & $\frac{3}{8}$ & $\frac{3}{8}$ & $\frac{1}{8}$ & 0 & 0 & 0 \\
11 & $\frac{1}{3}$ & $\frac{1}{3}$ & $\frac{1}{3}$ & 0 & 0 & 0 & 0 \\
21 & $\frac{5}{2}$ & $\frac{7}{2}$ & $\frac{7}{2}$ & $\frac{5}{2}$ & 0 & 0 & 0 \\
31 & $\frac{1}{8}$ & $\frac{1}{4}$ & $\frac{1}{4}$ & $\frac{1}{4}$ & $\frac{1}{8}$ & 0 & 0 \\
22 & $\frac{5}{32}$ & $\frac{5}{24}$ & $\frac{13}{48}$ & $\frac{5}{2} 4$ & $\frac{5}{32}$ & 0 & 0 \\
32 & $\frac{7}{64}$ & $\frac{11}{64}$ & $\frac{7}{32}$ & $\frac{7}{32}$ & $\frac{11}{64}$ & $\frac{7}{64}$ & 0 \\
33 & $\frac{7}{80}$ & $\frac{21}{16}$ & $\frac{3}{16}$ & $\frac{3}{16}$ & $\frac{3}{16}$ & $\frac{21}{160}$ & $\frac{7}{80}$ \\
\hline
\end{tabular}

Properties of $W\left(\rho_{1} \rho_{2}, \rho P\right)$ are

$$
\begin{gathered}
\rho_{1}+\rho_{2}=\rho+P, \quad W(00,00)=1, \\
W\left(\rho_{1} \rho_{2}, \rho P\right)=W\left(\rho_{2} \rho_{1}, \rho P\right)=W\left(\rho_{1} \rho_{2}, P \rho\right)=W\left(\rho_{2} \rho_{1}, P \rho\right), \\
W\left(\rho_{1} \rho_{2}, \rho P\right)=\frac{[\rho][P]}{\left[\rho_{1}\right]\left[\rho_{2}\right]} W\left(\rho P, \rho_{1} \rho_{2}\right), \\
\sum_{\rho} \sum_{P} W\left(\rho_{1} \rho_{2}, \rho P\right)=1, \\
W\left(\rho_{1} \rho_{2}, 0 \mathrm{P}\right)=\frac{\left(\rho_{1}+\rho_{2}+2\right) !}{\left(\rho_{1}+2\right) !\left(\rho_{2}+2\right) !} \frac{1}{2^{\rho_{1}+\rho_{2}-1}},
\end{gathered}
$$




$$
\begin{gathered}
W\left(\rho_{1} 0, \rho P\right)=\frac{1}{2^{\rho_{1}}} \frac{(\rho+P+2) !}{\rho ! P !\left(\rho_{1}+1\right)\left(\rho_{1}+2\right)}, \\
(\rho+1) W\left(\rho_{1} \rho_{2}, \rho+1 P\right)+(P+1) W\left(\rho_{1} \rho_{2}, \rho P+1\right) \\
=\rho_{1} W\left(\rho_{1}-1 \rho_{2}, \rho P\right)+\rho_{2} W\left(\rho_{1} \rho_{2}-1, \rho P\right), \\
\left(\rho_{1}+3\right) W\left(\rho_{1}+1 \rho_{2}, \rho P\right)+\left(\rho_{2}+3\right) W\left(\rho_{1} \rho_{2}+1, \rho P\right) \\
=(\rho+2) W\left(\rho_{1} \rho_{2}, \rho-1 P\right)+(P+2) W\left(\rho_{1} \rho_{2}, \rho P-1\right) .
\end{gathered}
$$

\section{Some numerical results}

In sect. 2, we gave formulae (2.16) and (2.18) for the separation energy of strongly bound nucleons. These formulae express the separation energy in terms of matrix elements $V_{n l}=\langle n l|\bar{v}| n l\rangle$ of the central part of the nucleon-nucleon interaction between states $|n l\rangle$ of relative motion of the interaction nucleons. If we wish to calculate separation energies using either phenomenological or "realistic" nucleonnucleon interactions we need numerical values of these matrix elements for relevant values of the harmonic oscillator radius parameter $b=(\hbar / M \omega)^{\frac{1}{2}}$. Several situations may occur.

(i) If the interaction is non-singular the matrix elements $V_{S, n l}$ for the singlet and triplet parts of the central interaction can be calculated by direct integration and then combined using eqs. (3.5a) and (4.7a,b) to give $V_{n l}$ or $V_{p}$. (The $S$ in $V_{S, n l}$ is the total spin of the two interaction nucleons; $S=0$ for a singlet interaction and $S=1$ for a triplet interaction,)

(ii) if the interaction is non-singular, numerical values of the matrix elements $V_{S, n l}$ may be given by other authors. It sometimes happens that the central, spin-orbit and tensor parts of the interaction are not separated. Instead values of the "reduced" matrix elements

$$
\langle n l S J|v| n l S J\rangle
$$

of the total nucleon-nucleon interaction (including spin-orbit and tensor parts) between states of relative motion of two nucleons characterized by the quantum numbers $n l$ with total spin $S$ and with $l$ and $S$ coupled to $J$ are tabulated. Values of the matrix elements $V_{S, n l}$ of the central part of the interaction can be extracted from these using the formula

$$
V_{S, n l}=\frac{1}{(2 S+1)(2 l+1)} \sum_{J}(2 J+1)\langle n l S J|v| n l S J\rangle .
$$

To see this we decouple the orbital and spin angular momenta $l$ and $S$ by writing the state $|n l S J M\rangle$ as a linear combination of states $\left|n l m_{l} m_{s}\right\rangle$ and Clebsch-Gordan coefficients. Using the orthogonality relations for Clebsch-Gordan coefficients, we get

$$
\sum_{J}(2 J+1)\langle n l S J|v| n l S J\rangle=\sum_{m_{l}} \sum_{m_{s}}\left\langle n l m_{l} S m_{s}|v| n l m_{l} S m_{s}\right\rangle .
$$


The right-hand side of eq. (5.2) contains an independent sum over the magnetic quantum numbers of $l$ and $S$. The sum will vanish for the spin-orbit and tensor parts of the potential $v$ because these transform like a vector or a tensor of rank 2 in orbital space and spin space. Thus only the central part will contribute. The matrix elements for this part of the interaction will be independent of the magnetic quant um numbers $m_{l}$ and $m_{s}$, and the right-hand side of eq. (5.2) will consist of $(2 /+1)(2 S+1)$ equal terms each with magnitude $V_{S, n l}$. This verifies eq. (5.1).

(iii) Over the past few years a number of "realistic" nucleon-nucleon interaction potentials have been determined from an analysis of nucleon-nucleon scattering. Some of these are non-singular and can be used directly for calculating the matrix elements $V_{n l}$. Others have an infinite repulsive core. Matrix elements calculated with these interactions are infinite and the whole theory based on the single-particle model breaks down. Various methods (reaction matrix methods, separation methods etc.) have been developed for replacing the interaction with its hard-core by some effective interaction which can then be used in shell-model type calculations. Such procedures sometimes lead to reaction matrix elements which can replace the potential energy matrix elements used in this paper. These reaction matrix elements have been tabulated for several hard core potentials by various authors and can be used to compute separation energies.

Table 2 contains matrix elements $V_{n l}$ computed from several phenomenological and realistic interactions with a value of the oscillator parameter $\hbar \omega=13.3 \mathrm{MeV}$ appropriate for ${ }^{16} \mathrm{O}$.

TABLE 2

Values of interaction matrix elements $V_{n t}$

\begin{tabular}{lllcc}
\hline$\rho$ & 0 & 1 & \multicolumn{2}{c}{2} \\
\hline$n l$ & 00 & 01 & 10 & 02 \\
\hline SW & -8.3 & 0.71 & -7.1 & -0.46 \\
BM & -8.4 & 0.05 & -7.4 & -0.47 \\
KK & -8.0 & 0.0 & -7.2 & 0.0 \\
A & -8.4 & 0.31 & -5.5 & 0.0 \\
\hline
\end{tabular}

The various interactions are described in the text.

The matrix elements for the interaction labelled SW have been computed by Shakin and Waghmare ${ }^{10}$ ) from the Yale ${ }^{11}$ ) potential using a unitary model-operator approach ${ }^{12}$ ). Those labelled BM have been calculated by Becker and Mackellar ${ }^{13}$ ) from the Hamada-Johnston potential ${ }^{14}$ ) using a reaction matrix method. The third group of matrix elements in table 2 have been calculated by Brink and Grypeos ${ }^{15}$ ) from the Kallio-Koltveit potential ${ }^{16}$ ) using a model-operator approach. The matrix elements $\mathrm{A}$ are calculated from a phenomenological central pseudo-potential defined 
in momentum space by

$$
\begin{gathered}
\left(\boldsymbol{k}|v| \boldsymbol{k}^{\prime}\right\rangle=A+B\left(\boldsymbol{k}^{2}+\boldsymbol{k}^{\prime 2}\right)+2 C \boldsymbol{k} \cdot \boldsymbol{k}^{\prime}, \\
A=1032 \mathrm{MeV} \cdot \mathrm{fm}^{3}, \quad B=317 \mathrm{MeV} \cdot \mathrm{fm}^{5}, \quad C=42.2 \mathrm{MeV} \cdot \mathrm{fm}^{5},
\end{gathered}
$$

where the parameters $A, B$ and $C$ have been determined to give the correct binding energy and density for nuclear matter and reasonable binding energies for light nuclei in the Hartree-Fock approximation. There are many interactions which give the correct binding energy and density for nuclear matter and reasonable binding energies for light nuclei. We selected $\mathrm{A}$ as an example of such an interaction in order to give a comparison between separation energies calculated with realistic and phenomenological interactions.

Separation energies of a 1 s or a 1 p neutron from ${ }^{16} \mathrm{O}$ can becomputed from the matrix elements in $V_{n l}$ in table 2 using eqs. (2.18), (2.19) and (4.7a). The numerical values are given in table 3. Proton separation energies must be corrected for the Coulomb energy which in ${ }^{16} \mathrm{O}$ gives a reduction of about $3.8 \mathrm{MeV}$ from the energies quoted in table 3 .

TABLE 3

Neutron separation energies from ${ }^{16} \mathrm{O}$ in $\mathrm{MeV}$

\begin{tabular}{rrrrr}
\hline Potential & SW & BM & KK & A \\
\hline Orbit 1 s & 47.0 & 52.4 & 50.0 & 50.7 \\
1 p & 21.3 & 25.4 & 23.0 & 22.2 \\
\hline
\end{tabular}

\section{Correlations to the SU(3) approximation}

In this section we investigate the corrections due to those parts of the central force which we neglected in the SU(3) approximation and due to neglect of the spin-orbit part of the nucleon-nucleon interaction.

In order to calculate the correction to the separation energy due to the parts of the central force which we neglected in the SU(3) approximation it is only necessary to calculate the matrix elements $\left\langle n_{2} l_{2}\left|W_{\rho_{1}}\right| n_{2} l_{2}\right\rangle$ from eq. (4.5) and to use eq. (2.16) for the separation energy. As an example we consider the separation energy of a $1 \mathrm{~s}$ nucleon $\left(\rho_{1}=0\right)$ from nuclei between ${ }^{16} \mathrm{O}$ and ${ }^{40} \mathrm{Ca}$. We assume that the levels in the open shell fill according to the $j j$ coupling scheme i.e. the first six protons (neutrons) go into the $\mathrm{d}_{\frac{3}{2}}$ level, the next two into the $\mathrm{s}_{\frac{1}{2}}$ level and the last four into the $\mathrm{d}_{\frac{3}{2}}$ level. The corrections to the $\mathrm{SU}(3)$ approximation depend only on $\left(V_{02}-V_{10}\right)$, i.e.

$$
\Delta \bar{E}_{0}=\left(V_{02}-V_{10}\right)\left(\frac{5}{3} \frac{N_{\mathrm{s}}}{2}-\frac{1}{32} \bar{N}_{\mathrm{d}}\right)
$$

in this case and are a maximum in ${ }^{28} \mathrm{Si}\left(\right.$ filled $\mathrm{d}_{\frac{5}{2}}$ shell $\Delta \bar{E}_{0}=\frac{3}{8}\left(V_{10}-V_{02}\right)$ ) and in ${ }^{32} \mathrm{~S}$ (filled $\mathrm{d}_{\frac{5}{2}}$ and $\mathrm{s}_{\frac{1}{2}}$ shells, $\Delta \bar{E}_{0}=-\frac{2}{8}\left(V_{10}-V_{02}\right)$ ). For the Kallio-Koltveit poten- 
tial $\left(V_{02}-V_{10}\right)=+7.1 \mathrm{MeV}$ hence the corrections to the $\mathrm{SU}(3)$ approximation in this case are less than $3 \mathrm{MeV}$ or less than $5 \%$.

The contribution of the spin-orbit forces to the average separation energy of a nucleon from the oscillator level $\rho_{1}$ is

$$
-\Delta\left(\bar{E}_{\rho_{1}}\right)_{\mathrm{so}}=\sum_{n_{2} l_{2} j_{2}} \bar{N}_{n_{2} l_{2} j_{2}}\left\langle n_{2} l_{2} j_{2}\left|W_{\rho_{1}}^{\mathrm{so}}\right| n_{2} l_{2} j_{2}\right\rangle
$$

and may be derived using methods similar to those discussed in subsect. 2.3. The matrix element of $W_{\rho_{1}}^{\text {so }}$ is defined in eq. (2.12) and $\bar{N}_{n_{2} l_{2} j_{2}}$ is the expectation value of the number of nucleons in the level $n_{2} l_{2} j_{2}$ in the state $|\Phi\rangle$. The contribution of the spin-orbit forces is difficult to evaluate exactly. However we can estimate their effect in the following way. If the spin-orbit force has a short range it can be shown by methods analogous to those used by Bell and Skyrme ${ }^{17}$ ) that

$$
\begin{aligned}
\left\langle n_{2} l_{2} j_{2}\left|W_{\rho_{1}}\right| n_{2} l_{2} j_{2}\right\rangle & =+U\left(l_{2}, \rho_{1}\right) l_{2} & \text { if } j_{2}=l_{2}+\frac{1}{2} \\
& =-U\left(l_{2}, \rho_{1}\right)\left(l_{2}+1\right) & \text { if } j_{2}=l_{2}-\frac{1}{2},
\end{aligned}
$$

where $U\left(I_{2}, \rho_{1}\right)$ depends on the radial wave functions of the states involved. We will assume that these coefficients are independent of the orbits, i.e.

$$
U\left(l_{2}, \rho_{1}\right)=U_{0}
$$

for the purpose of estimating the effect of the spin-orbit force. In order to obtain information about the magnitude of $U_{0}$ we note that the spin-orbit splitting of the level is

$$
\Delta E_{l_{2}}=\left(2 l_{2}+1\right) 4 \sum_{\rho^{\prime}}\left[\rho_{1}\right] U\left(l_{2}, \rho_{1}\right)=\left(2 l_{2}+1\right) U_{0} A
$$

where $A$ is the total number of nucleons in the nucleus.

If we apply eq. (6.5) to the ${ }^{16} \mathrm{O}$ nucleus, where the spin-orbit splitting is about 6 $\mathrm{MeV}$, we find that

$$
U_{0} \cong-0.12 \mathrm{MeV}
$$

The spin-orbit strength parameter $U_{0}$ is negative because the level $j_{2}=l_{2}+\frac{1}{2}$ lies below the level $j_{2}=l_{2}-\frac{1}{2}$.

Again we consider the contribution of the spin-orbit coupling to the separation energy of a 1 s nucleon from a nucleus between ${ }^{16} \mathrm{O}$ and ${ }^{40} \mathrm{Ca}$. The correction to the separation energy is

$$
-\Delta\left(E_{0}\right)_{\mathrm{so}}=\left(2 \bar{N}_{\mathrm{d}_{\frac{5}{2}}}-3 \bar{N}_{\mathrm{d}_{\frac{3}{2}}}\right) U_{0} .
$$

This is a maximum for the nuclei between ${ }^{28} \mathrm{Si}$ and ${ }^{32} \mathrm{~S}$ where it has the value

$$
-\Delta\left(E_{0}\right)_{\mathrm{so}}=24 U_{0}
$$

With $U_{0}$ given by eq. (6.6), the correction is positive with a magnitude of about 3 $\mathrm{MeV}$, again a correction of about $5 \%$. 
The corrections due to the central force and spin-orbit force tend to cancel between ${ }^{16} \mathrm{O}$ and ${ }^{28} \mathrm{Si}$ and add for nuclei between ${ }^{32} \mathrm{~S}$ and ${ }^{40} \mathrm{Ca}$. The maximum combined correction would be about $6 \mathrm{MeV}$ or $10 \%$ in ${ }^{31} \mathrm{~S}$. Both corrections vanish in the closed-shell nuclei ${ }^{16} \mathrm{O}$ and ${ }^{40} \mathrm{Ca}$.

It is a pleasure for both of us to acknowledge the cooperation of Professor M. Ageno and the group at the Istituto Superiore di Sanità, Rome, who brought the problem to our attention and who provided one of us (N.S.) with warm and most generous hospitality during a sabbatical year in Rome and the other (D.M.B.) with the opportunity to visit the Physics Laboratories and enjoy stimulating discussions of the (e, e'p) experiments. D.M.B. would like to thank the Physics Department of the University of Michigan for an invitation to attend its Summer Symposium on Nuclear Physics during which this work was completed. He is very grateful to the members of the Physics Department for their hospitality.

\section{Appendix 1}

Here we derive eq. (2.2)

$$
(T+V)-\eta_{\alpha}^{+}(T+V) \eta_{\alpha}=\langle\alpha|t| \alpha\rangle+W_{\alpha}+\eta_{\alpha} C+D \eta_{\alpha}^{+}-\eta_{\alpha}(T+V) \eta_{\alpha}^{+},
$$

where

$$
\begin{aligned}
T & =\sum_{\lambda_{\mu}} \sum_{\langle\lambda|t| \mu\rangle \eta_{\lambda}^{+} \eta_{\mu},} \\
V & =\frac{1}{4} \sum_{\kappa} \sum_{\lambda} \sum_{\mu} \sum_{\nu}\left\langle\kappa \hat{\lambda}\left|v_{A}\right| \mu \nu\right\rangle \eta_{\kappa}^{+} \eta_{\lambda}^{+} \eta_{\nu} \eta_{\mu} .
\end{aligned}
$$

We transform the operator products

$$
\eta_{\alpha}^{+} \eta_{\lambda}^{+} \eta_{\mu} \eta_{\alpha}, \quad \eta_{\alpha}^{+} \eta_{\kappa}^{+} \eta_{\lambda}^{+} \eta_{\nu} \eta_{\mu} \eta_{\alpha}
$$

which occur in the expansion of eq. (A.1) by using the anti-commutation relations to transfer the operator $\eta_{\alpha}^{+}$to the right of these products and the operator $\eta_{\alpha}$ to the left. As a first step we shift the operators $\eta_{\alpha}^{+}$and $\eta_{\alpha}$ into the centre of the products.

$$
\eta_{\alpha}^{+} \eta_{\lambda}^{+} \eta_{\mu} \eta_{\alpha}=\eta_{\lambda}^{+} \eta_{\alpha}^{+} \eta_{\alpha} \eta_{\mu}=\eta_{\lambda}^{+} \eta_{\mu}-\eta_{\lambda}^{+} \eta_{\alpha} \eta_{\alpha}^{+} \eta_{\mu}
$$

The last step in eq. (A.3) follows from the anti-commutation relation $\eta_{\alpha}^{+} \eta_{\alpha}=1-\eta_{\alpha} \eta_{\alpha}^{+}$. Similarly

$$
\eta_{\alpha}^{+} \eta_{\kappa}^{+} \eta_{\lambda}^{+} \eta_{v} \eta_{\mu} \eta_{\alpha}=\eta_{\kappa}^{+} \eta_{\lambda}^{+} \eta_{\nu} \eta_{\mu}-\eta_{\kappa}^{+} \eta_{\lambda}^{+} \eta_{\alpha} \eta_{\alpha}^{+} \eta_{v} \eta_{\mu}
$$

The transformation of eq. (A.3) is completed by using the anticommutation relations

$$
\eta_{\lambda}^{+} \eta_{\alpha}=\delta_{\alpha \lambda}-\eta_{\alpha} \eta_{\lambda}^{+}, \quad \eta_{\alpha}^{+} \eta_{\mu}=\delta_{\alpha \mu}-\eta_{\mu} \eta_{\alpha}^{+} .
$$

Substituting in eq. (A.3), we get

$$
\eta_{\alpha}^{+} \eta_{\lambda}^{+} \eta_{\mu} \eta_{\alpha}=\eta_{\lambda}^{+} \eta_{\mu}-\delta_{\alpha \lambda} \delta_{\alpha \mu}+\delta_{\alpha \lambda} \eta_{\mu} \eta_{\alpha}^{+}+\delta_{\alpha \mu} \eta_{\alpha} \eta_{\lambda}^{+}-\eta_{\alpha} \eta_{\lambda}^{+} \eta_{\mu} \eta_{\alpha}^{+} \text {. }
$$


To complete the transformation of eq. (A.4), we note that

$$
\begin{gathered}
\eta_{\kappa}^{+} \eta_{\lambda}^{+} \eta_{\alpha}=\delta_{\alpha \lambda} \eta_{\kappa}^{+}-\delta_{\alpha \kappa} \eta_{\lambda}^{+}+\eta_{\alpha} \eta_{\kappa}^{+} \eta_{\lambda}^{+}, \\
\eta_{\alpha}^{+} \eta_{\nu} \eta_{\mu}=\delta_{\alpha \nu} \eta_{\mu}-\delta_{\alpha \mu} \eta_{\nu}+\eta_{\nu} \eta_{\mu} \eta_{\alpha}^{+} .
\end{gathered}
$$

The second term on the right-hand side of eq. (A.4) is just the product of these factors. If we collect eqs. (A.5), (A.4) and (A.6) together and substitute into eqs. (A.2) for $T$ and $V$, we find

$$
(T+V)-\eta_{\alpha}^{+}(T+V) \eta_{\alpha}=B+\eta_{\alpha} C+D \eta_{\alpha}^{+}-\eta_{\alpha}(T+V) \eta_{\alpha}^{+}
$$

where the operators $B, C$ and $D$ contain no factors of $\eta_{x}$ or $\eta_{\alpha}^{+}$. We are interested only in the explicit form of $B$ which comes from the second term of eq. (A.5) and the first two terms of eq. (A.6)

$$
B=\sum_{\lambda} \sum_{\mu}\langle\lambda|t| \mu\rangle \delta_{\alpha \lambda} \delta_{\alpha \mu}+\frac{1}{4} \sum_{\kappa} \sum_{\lambda} \sum_{\mu} \sum_{v}\left\langle\kappa \lambda\left|v_{A}\right| \mu v\right\rangle\left(\delta_{\alpha \lambda} \eta_{\kappa}^{+}-\delta_{\alpha \kappa} \eta_{\lambda}^{+}\right)\left(\delta_{\alpha \nu} \eta_{\mu}-\delta_{\alpha \mu} \eta_{v}\right)
$$

The first term in eq. (A.8) can be evaluated immediately, and the second term simplified by interchanging summation indices so that each term contains a factor $\eta_{\kappa}^{+} \eta_{\mu}$ $B=\langle\alpha|t| \alpha\rangle+\frac{1}{4} \sum_{\kappa} \sum_{\mu}\left\{\left\langle\kappa \alpha\left|v_{A}\right| \mu \alpha\right\rangle-\left\langle\kappa \alpha\left|v_{A}\right| \alpha \mu\right\rangle+\left\langle\alpha \kappa\left|v_{A}\right| \alpha \mu\right\rangle-\left\langle\alpha \kappa\left|v_{A}\right| \mu \alpha\right\rangle\right\} \eta_{\kappa}^{+} \eta_{\mu}$.

Using the symmetry relations

$$
\left\langle\kappa \lambda\left|v_{A}\right| \mu \nu\right\rangle=-\left\langle\kappa \hat{\lambda}\left|v_{A}\right| v \mu\right\rangle=-\left\langle\hat{\lambda} k\left|v_{A}\right| \mu \nu\right\rangle=\left\langle\lambda \kappa\left|v_{A}\right| v \mu\right\rangle
$$

we get finally

$$
B=\langle\alpha|t| \alpha\rangle+\sum_{\kappa} \sum_{\mu}\left\langle\kappa \alpha\left|v_{A}\right| \mu \alpha\right\rangle \eta_{\kappa}^{+} \eta_{\mu}=\langle\alpha|t| \alpha\rangle+W_{\alpha},
$$

which proves eq. $(2.2)$.

\section{Appendix 2}

In this appendix we will prove eqs. (4.11)-(4.17), which show properties of the coefficients $W\left(\rho_{1} \rho_{2}, \rho P\right)$ stated in sect. 4. Eqs. (4.11) and (4.12) are symmetry relations which may be proved most easily by expressing them in terms of generalized TalmiMoshinsky transformation coefficients. We denote a two-nucleon orbital state by $\left|\rho_{1} u_{1} \rho_{2} u_{2}\right\rangle$ where $u_{1}, u_{2}$ stand for sets of quantum numbers labelling states in the oscillator shells $\rho_{1}$ and $\rho_{2}$. If we work with wave functions in a spherical representation then $u_{1}$ stands for the quantum numbers $n_{1} l_{1} m_{1}$ with $2 n_{1}+l_{1}=\rho_{1}$. Sometimes, however, it is useful to specify oscillator states in a rectangular representation. Then $u_{1}$ stands for the quantum numbers $n_{1 x} n_{1 y} n_{1 z}$ with $n_{1 x}+n_{1 y}+n_{1 z}=\rho_{1}$. Similarly we denote a two-nucleon state in the relative motion and centre-of-mass representation by $|\rho u P U\rangle$. The transformation between the two representations is

$$
\left|\rho_{1} u_{1} \rho_{2} u_{2}\right\rangle=\sum_{\rho u P U}|\rho u P U\rangle\left\langle\rho u P U \mid \rho_{1} u_{1} \rho_{2} u_{2}\right\rangle
$$


with $\rho_{1}+\rho_{2}=\rho+P$. Matrix elements of the projection operators $q_{\rho}$ can be expressed in terms of these transformation coefficients

$$
\begin{aligned}
& \left\langle\rho_{1} u_{1} \rho_{2} u_{2}\left|q_{\rho}\right| \rho_{1} u_{1} \rho_{2} u_{2}\right\rangle \\
& \quad=\sum_{P U \rho^{\prime} u^{\prime} \rho^{\prime \prime} u^{\prime \prime}}\left\langle\rho_{1} u_{1} \rho_{2} u_{2} \mid \rho^{\prime} u^{\prime} P U\right\rangle\left\langle\rho^{\prime} u^{\prime}\left|q_{\rho}\right| \rho^{\prime \prime} u^{\prime \prime}\right\rangle\left\langle\rho^{\prime \prime} u^{\prime \prime} P U \mid \rho_{1} u_{1} \rho_{2} u_{2}\right\rangle .
\end{aligned}
$$

Using the projection property of the operator $q_{\rho}$ of eq. (4.7b), this reduces to

$$
\left\langle\rho_{1} u_{1} \rho_{2} u_{2}\left|q_{\rho}\right| \rho_{1} u_{1} \rho_{2} u_{2}\right\rangle=\sum_{P U \rho u}\left|\left\langle\rho_{1} u_{1} \rho_{2} u_{2} \mid \rho u P U\right\rangle\right|^{2} .
$$

If we substitute this expression into eq. (4.9), we get

$$
W\left(\rho_{1} \rho_{2}, \rho P\right)=\frac{1}{\left[\rho_{1}\right]\left[\rho_{2}\right]} \sum_{u_{1} u_{2} u U}\left|\left\langle\rho_{1} u_{1} \rho_{2} u_{2} \mid \rho u P U\right\rangle\right|^{2} .
$$

The symmetry relations (4.11) and (4.12) follow directly from the symmetry relations for the Talmi-Moshinsky transformation coefficients

$$
\begin{aligned}
\left\langle\rho_{1} u_{1} \rho_{2} u_{2} \mid \rho u P U\right\rangle & =(-1)^{\rho}\left\langle\rho_{2} u_{2} \rho_{1} u_{1} \mid \rho u P U\right\rangle=(-1)^{\rho_{2}}\left\langle\rho_{1} u_{1} \rho_{2} u_{2} \mid P U \rho u\right\rangle \\
& =\left\langle\rho u P U \mid \rho_{2} u_{2} \rho_{1} u_{1}\right\rangle .
\end{aligned}
$$

The normalization condition for these coefficients,

$$
\sum_{\rho} \sum_{u} \sum_{P} \sum_{U}\left\langle\rho_{1} u_{1} \rho_{2} u_{2} \mid \rho u P U\right\rangle^{2}=1,
$$

leads to eq. (4.13):

$$
\sum_{\rho} \sum_{P} W\left(\rho_{2} \rho_{1}, \rho P\right)=1 .
$$

Eq. (4.14) gives an explicit formula for $W\left(\rho_{1} \rho_{2}, O P\right)$ which can be derived by writing eq. (4.9), with $\rho=0$, in the rectangular representation.

The operator $q_{0}$ which projects onto the ground state of relative motion is

$$
q_{0}=p_{0 x} p_{0 y} p_{0 z},
$$

where $p_{r x}$ is the projection onto the state with $r$ quanta excited in the $x$-degree of freedom in rectangular coordinates, and similarly for $p_{r y}$ and $p_{r z}$. The operator $q_{1}$ is

$$
q_{1}=p_{1 x} p_{0 y} p_{0 z}+p_{0 x} p_{1 y} p_{0 z}+p_{0 x} p_{0 y} p_{1 z},
$$

since each term projects onto a rectangular state with one quantum excited. Similar expressions can be written for $q_{2}$ etc.

The advantage of using rectangular coordinates rather than spherical ones is clearly that we can write matrix elements as a product of factors

$$
\begin{aligned}
& \left\langle n_{1 x} n_{1 y} n_{1 z} n_{2 x} n_{2 y} n_{2 z}\left|q_{0}\right| n_{1 x} n_{1 y} n_{1 z} n_{2 x} n_{2 y} n_{2 z}\right\rangle \\
& =\left\langle n_{1 x} n_{2 x}\left|p_{0 x}\right| n_{1 x} n_{2 x}\right\rangle\left\langle n_{1 y} n_{2 y}\left|p_{0 y}\right| n_{1 y} n_{2 y}\right\rangle\left\langle n_{1 z} n_{2 z}\left|p_{0 z}\right| n_{1 z} n_{2 z}\right\rangle
\end{aligned}
$$


Matrix elements of $q_{\rho}$ for $\rho \neq 0$ can be written as a sum of such products. The next problem is to calculate the effect of the projection operator $p_{0 x}$ on the state $\left|n_{1 x} n_{2 x}\right\rangle$. Consider the factored part of the two-particle wave function $\left|n_{1 x} n_{2 x}\right\rangle$ and re-write it as

$$
\left|n_{1 x} n_{2 x}\right\rangle=|m n\rangle \text {. }
$$

This wave function describes a state of excitation with $m$ quanta excited in the $x$-direction of the first oscillator and $n$ quanta in the $y$-direction for the second. It can be expressed in terms of the boson creation operators for quanta of a harmonic oscillator acting on the ground state $|0\rangle$ as follows

$$
|m n\rangle=\frac{1}{\sqrt{m ! n !}}\left(\alpha_{1}^{+}\right)^{m}\left(\alpha_{2}^{+}\right)^{n}|0\rangle
$$

where

$$
\begin{gathered}
{\left[\alpha_{1}^{+}, \alpha_{2}^{+}\right]=\left[\alpha_{1}, \alpha_{2}\right]=\left[\alpha_{1}, \alpha_{2}^{+}\right]=\left[\alpha_{2}, \alpha_{1}^{+}\right]=0,} \\
{\left[\alpha_{1}, \alpha_{1}^{+}\right]=\left[\alpha_{2}, \alpha_{2}^{+}\right]=1 .}
\end{gathered}
$$

(The factor $1 / \sqrt{m ! n !}$ is for normalization.) To transform the wave function, in this formalism, into a description of centre-of-mass and relative motion let

$$
A^{+}=\frac{1}{\sqrt{2}}\left(\alpha_{1}^{+}+\alpha_{2}^{+}\right), \quad a^{+}=\frac{1}{\sqrt{2}}\left(\alpha_{1}^{+}-\alpha_{2}^{+}\right) .
$$

Then:

$$
|m n\rangle=\frac{1}{\left(m ! n ! 2^{m+n}\right)^{\frac{1}{2}}}\left(A^{+}+a^{+}\right)^{m}\left(A^{+}-a^{+}\right)^{n}|0\rangle,
$$

and $p_{r}$ which projects out that part corresponding to a state with $r$ quanta excited in the relative motion can easily select that part. It is the term in the wave function which contains $\left(a^{+}\right)^{r}|0\rangle$. The result for $r=0$ is

$$
p_{0}|m n\rangle=\frac{1}{\left(m ! n ! 2^{m+n}\right)^{\frac{1}{2}}}\left(A^{+}\right)^{m+n}|0\rangle .
$$

Using the property

$$
\left\langle 0\left|A^{k}\left(A^{+}\right)^{l}\right| 0\right\rangle=k ! \delta_{k l},
$$

we can write down the matrix elements for $p_{0}$ as

$$
\left\langle m^{\prime} n^{\prime}\left|p_{0}\right| m n\right\rangle=\frac{(m+n) !}{2^{m+n}\left(m ! n ! m^{\prime} ! n^{\prime} !\right)^{\frac{1}{2}}} \delta\left(m+n-m^{\prime}-n^{\prime}\right) .
$$

Similar expressions can be obtained for the matrix elements of $p_{1}$ etc. We need only the diagonal matrix element of $p_{0}$

$$
\left\langle m n\left|p_{0}\right| m n\right\rangle=\frac{(m+n) !}{2^{m+n} m ! n !} .
$$


In the following we will use the fact that the matrix element $\left\langle m n\left|p_{0}\right| m n\right\rangle$ is the coefficient of $a^{m} b^{n}$ in the expansion of $\left[1-\frac{1}{2}(a+b)\right]^{-1}$ as a power series in $a$ and $b$. This can be verified by calculating the coefficient and comparing with eq. (A.16). We can now proceed to calculate the coefficient $W\left(\rho_{1} \rho_{2} . O P\right)$. According to its definition in eq. (4.9), we must sum the matrix element on the left-hand side of eq. (A.15) over all values of $n_{1 x}, n_{1 y}, n_{1 z}, n_{2 x}, n_{2 y}$ and $n_{2 z}$ subject to the restrictions

$$
\begin{aligned}
& n_{1 x}+n_{1 y}+n_{1 z}=\rho_{1}, \\
& n_{2 x}+n_{2 y}+n_{2 z}=\rho_{2},
\end{aligned}
$$

then divide by $\left[\rho_{1}\right]\left[\rho_{2}\right]$.

Each term in the sum is a product of three factors of eq. (A.15) and each factor is given by formula (A.16). It is a straightforward but not obvious task to see that the required sum is the coefficient of $a^{\rho_{1}} b^{\rho_{2}}$ in the expansion of $\left[1-\frac{1}{2}(a+b)\right]^{-3}$.

To see this we first consider the problem of calculating the sum

$$
w\left(\rho_{1} \rho_{2}\right)=\sum_{\substack{i+j+k=\rho_{1} \\ l+m+n=\rho_{2}}} f(i l) f(j m) f(k n),
$$

with

$$
f(i l)=\frac{1}{2^{i+l}} \frac{(i+l) !}{i ! l !} .
$$

The first step is to construct a generating function by multiplying both sides of eq. (A.17) by a factor $a^{\rho_{1}} b^{\rho_{2}}$ and summing over $\rho_{1}$ and $\rho_{2}$. We get

$$
\begin{aligned}
F(a, b) & =\sum_{\rho_{1}} \sum_{\rho_{2}} W\left(\rho_{1} \rho_{2}\right) a^{\rho_{1}} b^{\rho_{2}} \\
& =\sum_{i} \sum_{j} \sum_{k} \sum_{l} \sum_{m} \sum_{n} f(i l) f(j m) f(k n) a^{i+j+k} b^{l+m+n} \\
& =\left[\sum_{i} \sum_{l} f(i l) a^{i} b^{i}\right]^{3} \\
& =\left[\sum_{i} \sum_{l} \frac{(i+l) !}{i ! l !}\left(\frac{a}{2}\right)^{i}\left(\frac{b}{2}\right)^{l}\right]^{3} \\
& =\left[\sum_{n} \sum_{i} \frac{n !}{i !(m-i) !}\left(\frac{a}{2}\right)^{i}\left(\frac{b}{2}\right)^{n-i}\right]^{3} \\
& =\left[\sum_{n}\left(\frac{a+b}{2}\right)^{n}\right]^{3}=\frac{1}{\left(1-\frac{1}{2}(a+b)\right)^{3}} .
\end{aligned}
$$

Comparing eqs. (A.19) and (A.20) we see that $w\left(\rho_{1} \rho_{2}\right)$ is the coefficient of $a^{\rho_{1}} b^{\rho_{2}}$ in the expansion of $\left[1-\frac{1}{3}(a+b)\right]^{-3}$. Evaluating this coefficient using the binomial theorem we get

$$
w\left(\rho_{1} \rho_{2}\right)=\frac{\left(\rho_{1}+\rho_{2}+2\right) !}{\rho_{1} ! \rho_{2} ! 2^{\rho_{1}+\rho_{2}+1}} .
$$


We now return to the problem of evaluating the coefficient $W\left(\rho_{1} \rho_{2}, 0 P\right)$. If we write eq. (4.9) in the rectangular representation, we get

$$
\begin{aligned}
& W\left(\rho_{1} \rho_{2}, 0 P\right) \\
& =\frac{1}{\left[\rho_{1}\right]\left[\rho_{2}\right]} \sum_{\substack{n_{1 x}+n_{y}+n_{z}=\rho_{1} \\
n_{2 x}+n_{2 y}+n_{2 z}=\rho_{2}}}\left\langle n_{1 x} n_{1 y} n_{1 z} n_{2 x} n_{2 y} n_{2 z}\left|q_{0}\right| n_{1 x} n_{1 y} n_{1 z} n_{2 x} n_{2 y} n_{2 z}\right\rangle \\
& =\frac{1}{\left[\rho_{1}\right]\left[\rho_{2}\right]} \sum\left\langle n_{1 x} n_{2 x}\left|p_{0 x}\right| n_{1 x} n_{2 x}\right\rangle\left\langle n_{1 y} n_{2 y}\left|p_{0 y}\right| n_{1 y} n_{2 y}\right\rangle\left\langle n_{1 z} n_{2 z}\left|p_{0 z}\right| n_{1 z} n_{2 z}\right\rangle .
\end{aligned}
$$

Remembering that the matrix elements of $p_{0 x}, p_{0 y}$ and $p_{0 z}$ occurring in eq. (A.22) are given by eq. (A.16), we see that the sum in eq. (A.22) is identical to the sum in eq. (A.17). Hence

$$
W\left(\rho_{1} \rho_{2}, 0 P\right)=\frac{1}{\left[\rho_{1}\right]\left[\rho_{2}\right]} w\left(\rho_{1} \rho_{2}\right)=\frac{\left(\rho_{1}+\rho_{2}+2\right) !}{\left(\rho_{1}+2\right) !\left(\rho_{2}+2\right) ! 2^{\rho_{1}+\rho_{2}-1}},
$$

where we have used eq. (A.21) and $[\rho]=\frac{1}{2}(\rho+1)(\rho+2)$.

To derive the recursion formulae for $W\left(\rho_{1} \rho_{2} . \rho P\right)$ stated in sect. 4 , we write this coefficient as

$$
W\left(\rho_{1} \rho_{2}, \rho P\right)=\frac{1}{\left[\rho_{1}\right]\left[\rho_{2}\right]} \operatorname{Tr}\left(q_{\rho} q_{P}^{\mathrm{c}} q_{\rho_{1}}^{(1)} q_{\rho_{2}}^{(2)}\right),
$$

where $q_{\rho_{1}}^{(1)} q_{\rho_{2}}^{(2)}$ are the projection operators for the oscillator states of the first and second particles with total quantum numbers $\rho_{1}$ and $\rho_{2}$, respectively, and the operators $q_{\rho}$ and $q_{P}^{\mathrm{c}}$ are the analogous ones for the relative and centre-of-mass motion of the two particles. To see the equivalence between eqs. (B.16) and (4.9) we note that

$$
\begin{aligned}
\operatorname{Tr}\left(q_{\rho} q_{P}^{\mathrm{c}} q_{\rho_{1}}^{(1)} q_{\rho_{2}}^{(2)}\right)=\sum_{\rho_{1}} \sum_{u_{1}} \sum_{\rho_{2}} \sum_{u_{2}}\left\langle\rho_{1} u_{1} \rho_{2} u_{2}\left|q_{\rho} q_{P}^{\mathrm{c}} q_{\rho_{1}}^{(1)} q_{\rho_{2}}^{(2)}\right| \rho_{1} u_{1} \rho_{2} u_{2}\right\rangle \\
=\sum_{u_{1}} \sum_{u_{2}}\left\langle\rho_{1} u_{1} \rho_{2} u_{2}\left|q_{\rho} q_{P}^{\mathrm{c}}\right| \rho_{1} u_{1} \rho_{2} u_{2}\right\rangle
\end{aligned}
$$

because of the projection property of $q_{p_{1}}^{(1)}$ and $q_{p_{2}}^{(2)}$

Using

$$
q_{\rho_{1}}^{(1)} q_{\rho_{2}}^{(2)}\left|\rho_{1}^{\prime} u_{1} \rho_{2}^{\prime} u_{2}\right\rangle=\left|\rho_{1} u_{1} \rho_{2} u_{2}\right\rangle \delta_{\rho_{1} \rho_{2}^{\prime}} \delta_{\rho_{2} \rho_{2}^{\prime}} .
$$

$$
\left\langle\rho_{1} u_{1} \rho_{2} u_{2}\left|q_{\rho} q_{P}^{\mathrm{c}}\right| \rho_{1} u_{1} \rho_{2} u_{2}\right\rangle=\delta\left(P, \rho_{1}+\rho_{2}-\rho\right)\left\langle\rho_{1} u_{1} \rho_{2} u_{2}\left|q_{\rho}\right| \rho_{1} u_{1} \rho_{2} u_{2}\right\rangle,
$$

eq. (A.25) reduces to eq. (4.9). We need the recursion relations for $q_{\rho}$ :

$$
\begin{gathered}
a_{x}^{+} q_{\rho} a_{x}+a_{y}^{+} q_{\rho} a_{y}+a_{z}^{+} q_{\rho} a_{z}=\sum_{\gamma} a_{\gamma}^{+} q_{\rho} a_{\gamma}=(\rho+1) q_{\rho+1}, \\
\sum_{\gamma} a_{\gamma} q_{\rho} a_{\gamma}^{+}=(\rho+2) q_{\rho-1},
\end{gathered}
$$


where $a_{\gamma}^{+}$and $a_{\gamma}$ are creation and annihilation operators for oscillator quanta in the $x, y$ and $z$ directions. There are similar recursion formulae for the centre-of-mass projection operator $q_{P}^{\mathrm{c}}$ and for $q_{\rho_{1}}^{(1)}$ and $q_{\rho_{2}}^{(2)}$. These relations give

$$
\begin{aligned}
(\rho+2) q_{\rho-1} q_{P}^{\mathrm{c}}+(P+2) q_{\rho} q_{P-1}^{\mathrm{c}} & =\sum_{\gamma} a_{\gamma} q_{\rho} q_{P}^{\mathrm{c}} a_{\gamma}^{+}+\sum_{\gamma} A_{\gamma} q_{\rho} q_{P}^{\mathrm{c}} A_{\gamma}^{+} \\
& =\sum_{\gamma} \alpha_{1 \gamma} q_{\rho} q_{P}^{\mathrm{c}} \alpha_{1 \gamma}^{+}+\sum_{\gamma} \alpha_{2 \gamma} q_{\rho} q_{P}^{\mathrm{c}} \alpha_{2 \gamma}^{+} .
\end{aligned}
$$

The last line is obtained by substituting

$$
a_{1 \gamma}=\frac{1}{\sqrt{ } 2}\left(\alpha_{1 \gamma}-\alpha_{2 \gamma}\right), \quad A_{\gamma}=\frac{1}{\sqrt{ } 2}\left(\alpha_{1 \gamma}+\alpha_{2 \gamma}\right)
$$

in eq. (A.28). The cross terms cancel. From the property that the trace of a product of operators is invariant for a cyclic permutation of the operators in the product and using eq. (A.26), we get

$$
\begin{aligned}
\operatorname{Tr}\left\{\left[(\rho+2) q_{\rho-1} q_{P}^{\mathrm{c}}+(P+2) q_{\rho}^{\mathrm{c}} q_{P-1}\right] q_{\rho_{1}}^{(1)} q_{\rho_{2}}^{(2)}\right\} \\
=\operatorname{Tr}\left\{q_{\rho} q_{P}^{\mathrm{c}}\left[\left(\rho_{1}+1\right) q_{\rho_{1}+1}^{(1)} q_{\rho_{2}}^{(2)}+\left(\rho_{2}+1\right) q_{\rho_{1}}^{(1)} q_{\rho_{2}+1}^{(2)}\right]\right\} .
\end{aligned}
$$

Combining eq. (A.29) with (A.25) we get finally

$$
\begin{aligned}
(\rho+2) W\left(\rho_{1} \rho_{2}, \rho-1 P\right)+(P+2) W & \left(\rho_{1} \rho_{2}, \rho P-1\right) \\
= & \left(\rho_{1}+3\right) W\left(\rho_{1}+1 \rho_{2}, \rho P\right)+\left(\rho_{2}+3\right) W\left(\rho_{1} \rho_{2}+1, \rho P\right) .
\end{aligned}
$$

This is relation (4.17) of sect. 4. Relation (4.18) is obtained in a similar way.

\section{References}

1) D. M. Brink and N. Sherman, Phys. Rev. Lett. 14 (1965) 393

2) U. Amaldi et al., Phys. Rev. Lett. 13 (1964) 341

3) L. A. Sliv and B. A. Vochok, JETP (Sov. Phys.) 9 (1959) 374

4) S. A. Mozskowski, Encyclopedia of physics, Vol. 39, ed. by S. Flügge (Springer-Verlag, Berlin, 1957) p. 431

5) T. H. R. Skyrme, Nuclear Physics 9 (1959) 615

6) R. Muthukrishnan and M. Baranger, Phys. Lett. 18 (1965) 160;

K. T. R. Davies, S. J. Krieger and M. Baranger, Nuclear Physics 84 (1966) 545

7) S. A. Moszkowski and B. L. Scott, Nuclear Physics 40 (1962) 593

8) M. Moskinsky and T. A. Brody, Tables of transformation brackets (Monografias del Instituto de Física, Universidad de Mexico, Mexico, D.F. 1960)

9) D. M. Brink, Nuclear Physics 40 (1963) 593

10) C. Shakin and Y. R. Waghmare, Phys. Rev. Lett. 16 (1966) 403

11) K. E. Lassila et al., Phys. Rev. 126 (1962) 881

12) F. Villars, Proc. Enrico Fermi Int. School of Physics, Course XXIII 1961 (Academic Press, New York, 1963);

J. da Providencia and C. M. Shakin, Ann. of Phys. 30 (1964) 95

13) R. L. Becker and A. D. Mackellar, Phys. Lett. 21 (1966) 201

14) T. Hamada and I. D. Johnston, Nuclear Physics 34 (1962) 383

15) D. M. Brink and M. Grypeos, private communication

16) A. Kallis and K. Kolltveit, Nuclear Physics 53 (1964) 87

17) J. S. Bell and T. H. R. Skyrme, Phil. Mag. 1 (1956) 1055 